separated. $\mathrm{CSF}$ pH were somewhat lowered but in narrow range between 7.2: and 7.35. Homeostasis of $\mathrm{CSF} \mathrm{pH}$ was seemingly maintained, when $\mathrm{HCO}_{3}^{-}$defici was compensated by decreased $\mathrm{pCO}_{2}$. If the metabolic acidosis of CSF wa: associated with insufficient hyperventilation, $\mathrm{CSF} \mathrm{pH}$ was consequently lowered Increment of lactate in CSF, which was varified immediately after trauma, corre.

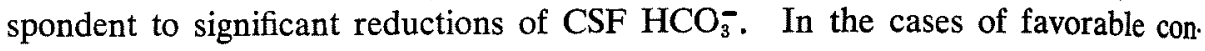

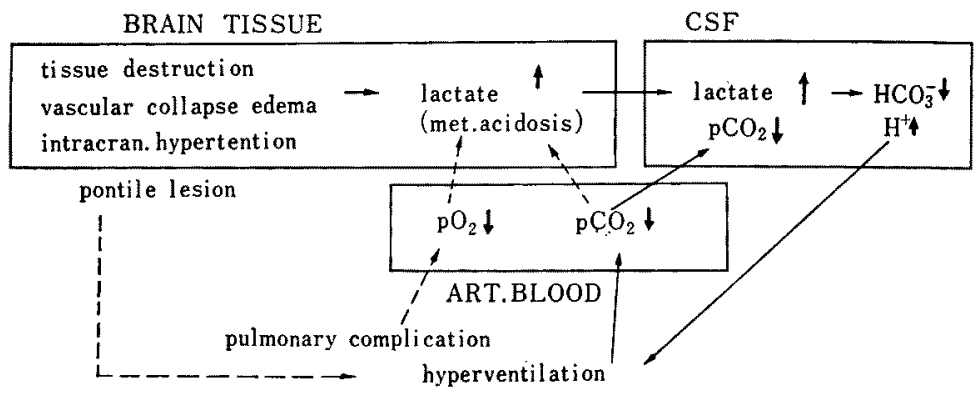

valescence, both lactate and $\mathrm{HCO}_{3}^{-}$in $\mathrm{CSF}$ were normalized simultaneously within a week of course.

Acid-base status of CSF, especially $\mathrm{HCO}_{3}^{-}$concentration, is a good indicator of metabolic disturbance in the brain tissue which is less influenced by blood than other indexes such as $\mathrm{H}^{+}$and $\mathrm{pCO}_{2}$. Severe hyperventilation may aggravate the metabolic acidosis conversely. Here, the validity of intrathecal administration of buffer agents is consecutively to be estimated.

\title{
1-4. Polygraphic Study as a Prognostic Criterion in Post-traumatic Coma
}

\author{
Yasuharu Kodama and Shigeaki Matsuoka \\ 1st Department of Surgery, School of Medicine, Kumamoto University \\ Giichi MiUra, Moritaka Miyagawa and Katsuyuki Otsuka \\ Department of Neurosurgery, Saiseikai Kumamoto Hospital
}

For the clinical evolution of post-traumatic comatose patients serial polygraphic recordings were taken night and day, which were EEG, chin-EMG, both eye movements and ECG and in some cases with the temperature of the tympanum or the plethysmogram.

Our comatose subjects was composed of 23 cases with acute head injury, in which 20 cases had operative treatments. This study was helped by 10 cases of post-traumatic prolonged coma, 4 coma cases of brain tumor and one come 
:ase with ruptured cerebral aneurysm.

This examination revealed that the typical polygraphic sleep patterns and organized circadian activity was shown in 13 patients with favourable prognosis and on the other hand, nocturnal unchanged "biphasic" or "monophasic" activity was seen in fatal cases.

In our 23 cases the presence of the typical sleep patterns or organized circadian rhythm suggests a favorable prognosis in post-traumatic comatose state and seems the very valid neurophysiological criterion for prognosis.

To judge the prognosis of comatose patients after head injury, to take the nocturnal and diurnal polygraphic recording are useful and recommended.

\title{
1-5. $\mathrm{PaCO}_{2}$ and $\mathrm{PaO}_{2}$ in Acute Stage of Patient with Severe Head Injury and Pulmonary Complication
}

\author{
Tohru Watanabe \\ Division of Neurosurgery, Matsue Red Cross Hospital \\ Tetsumi Mitani and Koh Namba \\ Division of Neurosurgery, Kokura Memorial Hospital \\ Yuzo Fusita \\ Department of Neurosurgery, Kyoto University School of Medicine
}

Head injury may be associated with thoracic injury or may occur to those who have preexisting lung disease. It has been stressed that in a comatose patient, the airway is apt to obstruct and product hypoxemia and hypercapnia. $\mathrm{PaCO}_{2}$ and $\mathrm{PaO}_{2}$ in acute stage were studied of 8 patients having severe head injury and the associated pulmonary complication.

Five cases had positive $\mathrm{x}$-ray findings such as diffuse veiling or pnemothrax, and two had chronic pulmonary diseases. In a patient, in whom examinations failed to reveal serious signs, the hypoxemia had continued to aggravate until an assisted repiration was started.

All were kept under oxygen inhalation and prevented from airway obstruc'ion with endotracheal tubing or tracheotomy. None had a controlled respiration.

$\mathrm{PaCO}_{2}$ was decreased or within normal range in all except one who had lad pulmonary tuberculosis. $\mathrm{PaO}_{2}$ was decreased without exceptions, but con;omitant increase of $\mathrm{PaCO}_{2}$ (hypercapnia) never occurred to 7 cases. Effective flleviation of the hypoxemia was achieved first with mechanical assisted respiration. 